\title{
SEPARABILITY OF METRIC SPACES
}

\author{
BY \\ PRABIR ROY
}

\section{Part I. Introduction AND Preliminaries}

A topological space $S$ is separable means that some countable subset of $S$ is dense in $S$. A subset $T$ of a topological space is separable means that $T$ has a countable subset which is dense in $T$. In view of the importance of this property, it is natural to inquire about conditions under which a space is separable. In this paper we shall discuss such conditions for metric spaces only $\left({ }^{1}\right)$.

The following theorems give a few of the known conditions for separability

TheOREM 1.1 (AleXANDROFF $[1]\left({ }^{2}\right)$ ). If a connected metric space $S$ is locally separable $\left({ }^{3}\right)$, then $S$ is separable.

THEOREM 1.2 (JONES [3]). If a connected, locally connected $\left({ }^{4}\right)$, metric space $S$ is locally peripherally separable $\left({ }^{5}\right)$, then $S$ is separable.

THEOREM $1.3\left(\operatorname{JONES}\left({ }^{6}\right)\right)$. If a connected, locally peripherally connected $\left({ }^{7}\right)$, metric space $S$ is locally peripherally separable, then $S$ is separable.

Theorem 1.3 is a special case of Theorem 1.2, because every connected and locally peripherally connected space is locally connected. However, we shall see that the particular phrasing of Theorem 1.3 leads to an interesting generalization.

As a measure of the sharpness of the above conditions we have the following example.

Received by the editors January 23, 1968 and, in revised form, May 7, 1969.

(1) For separability of spaces not necessarily metric, see [2].

( $\left.{ }^{2}\right)$ Numbers in brackets refer to the bibliography.

$\left.{ }^{3}\right)$ A space is locally separable at a point $p$ means that if $U$ is an open set containing $p$ then $U$ has an open subset $V$ which contains $p$ and which is separable. A space is locally separable means that the space is locally separable at each point in the space. Henceforth, if $p$ is a local property then a space will be understood to have that property if it has it at each of its points.

( $\left.{ }^{4}\right)$ A space is locally connected at a point $p$ means that if $U$ is an open set containing $p$ then $U$ has an open subset $V$ which contains $p$ and which is connected.

${ }^{(5)}$ A space is locally peripherally separable at a point $p$ means that if $U$ is an open set containing $p$ then $U$ has an open subset $V$ which contains $p$ and whose boundary is separable.

$\left.{ }^{(}\right)$This is an unpublished theorem of F. B. Jones.

${ }^{(7)}$ A space is locally peripherally connected at a point $p$ means that if $U$ is an open set containing $p$ then $U$ has an open subset which contains $p$ and whose boundary is connected.

Copyright (C) 1970, American Mathematical Society 
TheOREM 1.4 (TREYBIG [10]). There is a connected metric space $S$ such that

(1) $S$ is semi-locally-connected $\left({ }^{8}\right)$,

(2) $S$ is locally peripherally separable,

(3) every pair of points in $S$ belongs to a separable continuum $\left({ }^{9}\right)$,

(4) if $T=\{p \mid S$ is not locally separable at $p\}$, then $T$ is separable,

(5) but $S$ is not separable.

The purpose of this paper is to display what is perhaps the main theme underlying the above results; that is, the separability of certain subsets together with the interplay between various connectedness conditions and existence of appropriate separating $\left({ }^{10}\right)$ sets lead to the separability of the whole space.

In Part II, we give an example of a space which includes Theorem 1.4 and which provides counterexamples to many plausible conjectures in this area. This is also an example of a locally peripherally compact, connected, metrizable space which is not separable which answers a question in [4, p. 122].

In Part III, we investigate a question that arises from Theorem 1.4; namely, suppose that the hypothesis of Theorem 1.1 is satisfied by all points except those in a set $T$, what conditions on $T$ will guarantee the separability of the whole space?

Theorem 1.4 states that separability of $T$ is not enough. Also we consider the case where, in the above question, the hypothesis of Theorem 1.1 is replaced by the hypothesis of Theorem 1.2.

Part IV contains conditions for separability parallel to those in Theorems 1.1, 1.2, and 1.3. First we consider spaces which are not necessarily locally connected and give conditions for separability which avoid local properties as much as possible. In the latter part of this section we restrict our attention to the locally connected spaces. Here we find a generalization of Theorem 1.2 which may be the best possible along these lines.

The relations between separability as described in the first paragraph and some of the stronger forms of separability are given by the following theorems. We shall use these well-known properties throughout the rest ${ }^{*}$ of the paper.

THeOREM 1.5. A separable metric space is hereditarily separable $\left({ }^{11}\right)$.

THEOREM 1.6. For a metric space the following two statements are equivalent. (i) The space is separable. (ii) The space is perfectly separable $\left({ }^{12}\right)$.

$\left({ }^{8}\right)$ A space $S$ is semi-locally-connected at a point $p$ means that if $U$ is an open set containing $p$ then $U$ has an open subset $V$ which contains $p$ and $S-U$ intersects at most finitely many components of $S-V$.

( ${ }^{9}$ ) A continuum is a closed and connected point-set.

(10) A set $T$ is a separating set of a set $S$ means that $T$ is a subset of $S, S$ is connected, but $S-T$ is not connected.

(11) A space is hereditarily separable means that every subset of the space is separable.

(12) A space is perfectly separable means that every collection of open sets, whose sum is the whole space, has a countable subcollection whose sum is also the whole space. 
It should be noted that, by Theorem 1.5, the weaker separability conditions in the hypotheses in many of the theorems would follow from the separability of the space. Hence, for the most part, these theorems could be readily but more awkwardly stated in the "necessary and sufficient" form.

This paper is essentially a doctorate dissertation [8] written under the direction of Professor F. B. Jones at the University of North Carolina, Chapel Hill. The author expresses his deep appreciation to his teachers, F. B. Jones and J. S. Mac Nerney.

\section{PART II. AN EXAMPLe}

1. The description of the space. We denote by $\Gamma$ the space that will be described here. First we give a rough description of $\Gamma$. We shall use the three figures given in this chapter. The legends for these figures may be ignored for the present.

A. Take a rectangle plus its interior, with height 4 units and width 1 unit, and remove the bottom edge of this rectangle.

B. Take an infinite sequence of congruent rectangles converging to the first rectangle in the manner shown in Figure I, only the points in a subrectangle, with height 1 unit and width 1 unit, of the first rectangle being limit points of the infinite sequence of rectangles. One can visualize the first rectangle as standing upright, in the 3-dimensional Euclidean space, on its removed edge, and the sequence of rectangles, congruent and parallel to the first one but lowered by 3 units, converging to the first one.

C. Now repeat the process, using each of the rectangles in the sequence of rectangles as the first rectangle and for it getting a new sequence of rectangles.

D. Continue this process a countable number of times.

E. For each sequence $s$ of positive integers, with $s(1)=1$, we have an unique way of descending down this pyramid of rectangles; start with the topmost rectangle, go to the $s(2)$ th one in the sequence of rectangles converging to the first one, go to the $s(3)$ th rectangle in the sequence of rectangles converging to the one chosen immediately before, and so on. See Figure III. So we can imagine that we put a point at the end of every path, going one level at a time, down the pyramid of rectangles, and label this point by the sequence of positive integers describing the path. Let us call these points the base points.

F. Make uncountably many copies of the whole structure described in A-E, one for each number in $(0,1)$.

G. Put these copies together along their base points, identifying each base point in one of these copies with its congruent base point in the rest of the copies. Thus we can think of each copy as a leaf of a book, with these leaves being held together by the base points acting as a hinge.

H. Now we describe a special "leaf" (corresponding to the number 1 in the later description). Consider Figure II, and imagine that each of the lower rectangles is attached to the higher rectangle along the horizontal line with height 1 unit of the 
higher rectangle and the upper edge of the lower rectangles. Now proceed as in C and $\mathrm{D}$.

J. Define the base points for this structure as in E.

K. Attach this particular leaf to the others along its base points as described in $\mathbf{G}$.

The topology for $\Gamma$ is the obvious one suggested by the above description. It should be noted that the "leaves" are "far" from each other, except of course at their base points. Also, for a rectangle and a sequence of rectangles converging to the first one (see Figure I), the lower ends of the converging rectangles are "far" from each other.

Now we shall describe the points in $\Gamma$ more carefully. Let $p$ be a point in $\Gamma$ if either (i) $p$ is an ordered triplet $\left\{p_{1} ; p_{2} ; p_{3}\right\}$ where

(2.1) $p_{1}$ is an ordered real number pair $\left\{p_{11}, p_{12}\right\}$ such that $0<p_{11} \leqq 1$, and $p_{12}$ is a positive integer,

(2.2) $p_{2}$ is a sequence $\left\{p_{2}(1), p_{2}(2), \ldots, p_{2}\left(p_{12}\right)\right\}$ of positive integers with $p_{2}(1)=1$, and

(2.3) $p_{3}$ is a complex number such that $0 \leqq \operatorname{Re}\left(p_{3}\right) \leqq 1$, and $0<\operatorname{Im}\left(p_{3}\right) \leqq 4$, where $\operatorname{Im}\left(p_{3}\right)$ is allowed to equal 4 only in case $p_{11} \neq 1$, or (ii) $p$ is an ordered pair $\left\{p_{1} ; p_{2}\right\}$ where

(2.4) $p_{1}$ is an ordered pair $\left\{p_{11}, p_{12}\right\}$ such that $p_{11}=0$, and $p_{12}=\infty$, and

(2.5) $p_{2}$ is an infinite simple sequence $\left\{p_{2}(1), p_{2}(2), \ldots\right\}$ of positive integers with $p_{2}(1)=1$.

Let $U$ be a region $\left({ }^{13}\right)$ only in case there is a point $p$ and a positive integer $n$ such that

(2.6) if $p_{11}>0$ and $\operatorname{Im}\left(p_{3}\right)>1$, then

$$
U=\left\{q\left|q_{1}=p_{1} ; q_{2}=p_{2} ;\right| q_{3}-p_{3} \mid<\min \left\{1 / n, \operatorname{Im}\left(p_{3}\right)-1\right\}\right\},
$$

(2.7) if $0<p_{11}<1$ and $\operatorname{Im}\left(p_{3}\right) \leqq 1$, then

$$
\begin{aligned}
& U=\{\left\{\left|q_{1}=p_{1} ; q_{2}=p_{2} ;\right| q_{3}-p_{3} \mid<1 / n\right\} \\
&+\left\{q \mid q_{11}=p_{11} ; q_{12}=p_{12}+1 ; q_{2}(j)=p_{2}(j) \text { for } j=1, \ldots, p_{12} ;\right. \\
&\left.q_{2}\left(q_{12}\right) \geqq n ;\left|q_{3}-\left(p_{3}+3\right)\right|<1 / n\right\},
\end{aligned}
$$

(2.8) if $p_{11}=1$ and $\operatorname{Im}\left(p_{3}\right)=1$, then

$$
\begin{aligned}
U= & \left\{q\left|q_{1}=p_{1} ; q_{2}=p_{2} ;\right| q_{3}-p_{3} \mid<1 / n\right\} \\
& +\left\{q \mid q_{11}=p_{11} ; q_{12}=p_{12}+1 ; q_{2}(j)=p_{2}(j) \text { for } j=1, \ldots, p_{12} ;\right. \\
& \left.\left|q_{3}-\left(p_{3}+3\right)\right|<1 / n\right\},
\end{aligned}
$$

(2.9) if $p_{11}=1$ and $\operatorname{Im}\left(p_{3}\right)<1$, then

$$
\begin{aligned}
U=\left\{q\left|q_{1}=p_{1} ; q_{2}=p_{2} ;\right| q_{3}-p_{3} \mid<\min \left\{1 / n, 1-\operatorname{Im}\left(p_{3}\right)\right\}\right\} \\
+\left\{q \mid q_{11}=p_{11} ; q_{12}=p_{12}+1 ; q_{2}(j)=p_{2}(j) \text { for } j=1, \ldots, p_{12} ;\right. \\
\left.q_{2}\left(q_{12}\right) \geqq n ;\left|q_{3}-\left(p_{3}+3\right)\right|<\min \left\{1 / n, 1-\operatorname{Im}\left(p_{3}\right)\right\}\right\},
\end{aligned}
$$

(13) A region is an element of a basis for the topology. 
(2.10) if $p_{11}=0$, then

$$
U=\left\{q \mid q_{12} \geqq n ; q_{2}(j)=p_{2}(j) \text { for } j=1, \ldots, n\right\} .
$$

Such an $U$ will be called the open disc with center $p$ and radius $1 / n$, and will be denoted by $R_{1 / n}(p)$.

Let $G$ be a simple sequence of collections of regions such that if $n$ is a positive integer then a region $U$ is in $G_{n}$ only in case the radius of $U$ is less than or equal to $1 / n$.

The following figures are intended to elucidate the space $\Gamma$.

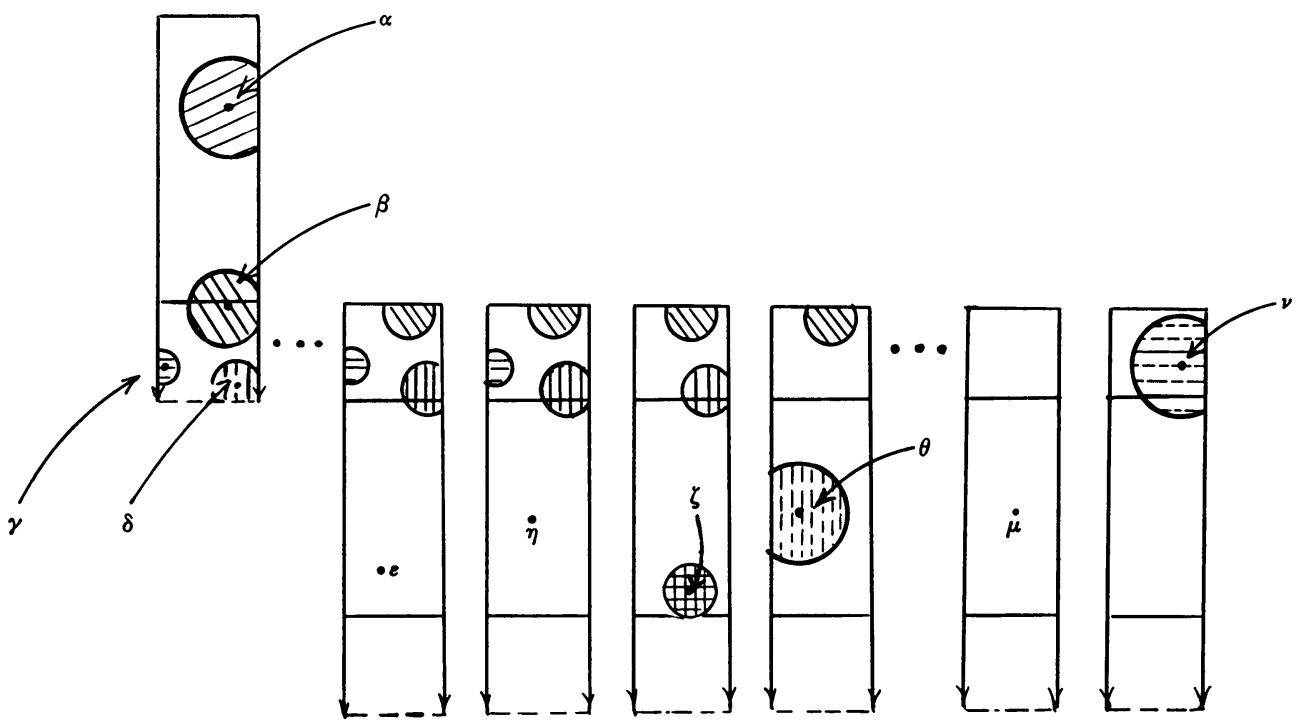

FIGURE I

Legend for Figure I. a. The dashed lines and the points indicated by curved arrowheads are not in the space. We suppose that each of $m$ and $n$ is a positive integer, $r$ is a number such that $0<r<1$, and $\{s(1)=1, \ldots, s(m)\}$ is a sequence of positive integers.

b. $\alpha=\left\{\left\{\alpha_{11}=r, \alpha_{12}=m\right\} ;\left\{\alpha_{2}=s\right\} ; 3 / 4+3 i\right\}$,

$\beta=\left\{\{r, m\} ;\left\{\beta_{2}=s\right\} ; 2 / 3+i\right\}$,

$\gamma=\left\{\{r, m\} ;\left\{\gamma_{2}=s\right\} ; i / 3\right\}$,

$\delta=\left\{\{r, m\} ;\left\{\delta_{2}=s\right\} ; 3 / 4+i / 8\right\}$,

$\varepsilon=\left\{\{r, m+1\} ;\left\{\varepsilon_{2}(1)=s(1), \ldots, \varepsilon_{2}(m)=s(m), \varepsilon_{2}(m+1)=n+3\right\} ; 1 / 3+3 i / 2\right\}$,

$\eta=\left\{\{r, m+1\} ;\left\{\eta_{2}(1)=s(1), \ldots, \eta_{2}(m)=s(m), \eta_{2}(m+1)=n+2\right\} ; 1 / 3+2 i\right\}$,

$\zeta=\left\{\{r, m+1\} ;\left\{s(1), \ldots, s(m), \zeta_{2}(m+1)=n+1\right\} ; 1 / 2+4 i / 3\right\}$,

$\theta=\{\{r, m+1\} ;\{s(1), \ldots, s(m), n\} ; 1 / 4+2 i\}$,

$\mu=\{\{r, m+1\} ;\{s(1), \ldots, s(m), 2\} ; 1 / 2+9 i / 4\}$,

$\nu=\{\{r, m+1\} ;\{s(1), \ldots, s(m), 1\} ; 3 / 4+10 i / 3\}$. 
c. $\square$ indicates $R_{1 / 2}(\alpha)$, in scale.

$\square$ indicates $R_{1 / n}(\beta)$ (cannot be in scale).

indicates $R_{1 / n+1}(\delta)$ (cannot be in scale).

$\square$ indicates $R_{1 / n+2}(\gamma)$ (cannot be in scale).

$\square$ indicates $R_{1 / 2}(\zeta)$, in scale.

$\square$ indicates $R_{1 / 2}(\theta)$, in scale.

$\square$ indicates $R_{1 / 2}(v)$, in scale.

Figure I illustrates a basic unit of construction (corresponding to the sequence $s$ ) in a "leaf" (corresponding to the number $r \neq 1$ ) in the space $\Gamma$. The "leaf" is $\left\{p \mid p_{11}=r\right\}$.

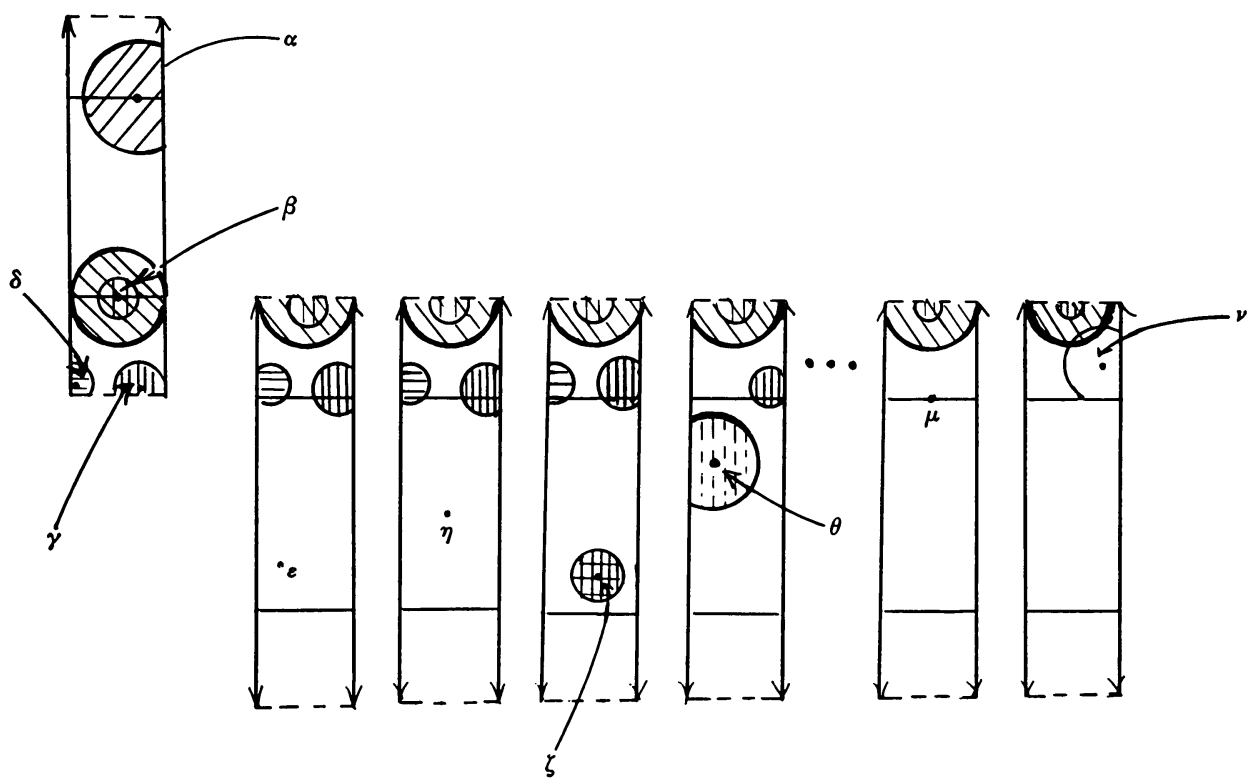

FIGURE II

Legend for Figure II. a. The dashed lines and the points indicated by curved arrowheads are not in the space. We suppose that each of $m$ and $n$ is a positive integer and $\{s(1)=1, s(2), \ldots, s(m)\}$ is a sequence of positive integers.

b. $\alpha=\left\{\left\{\alpha_{11}=1 ; \alpha_{12}=m\right\} ;\left\{\alpha_{2}=s\right\} ; 3 / 4+3 i\right\}$,

$\beta=\left\{\{1, m\} ;\left\{\beta_{2}=s\right\} ; 1 / 2+i\right\}$,

$\gamma=\left\{\{1, m\} ;\left\{\gamma_{2}=s\right\} ; 3 / 4+i / 4\right\}$,

$\delta=\left\{\{1, m\} ;\left\{\delta_{2}=s\right\} ; 1 / 4+i / 4\right\}$,

$\varepsilon=\left\{\{1, m+1\} ;\left\{\varepsilon_{2}(1)=s(1), \ldots \varepsilon_{2}(m)=s(m), \varepsilon_{2}(m+1)=n+3\right\} ; 1 / 3+3 i / 2\right\}$,

$\eta=\{\{1, m+1\} ;\{s(1), \ldots, s(m), n+2\} ; 2 / 3+2 i\}$,

$\zeta=\{\{1, m+1\} ;\{s(1), \ldots, s(m), n+1\} ; 1 / 2+4 i / 3\}$,

$\theta=\{\{1, m+1\} ;\{s(1), \ldots, s(m), n\} ; 1 / 3+7 i / 3\}$,

$\mu=\{\{1, m+1\} ;\{s(1), \ldots, s(m), 2\} ; 1 / 2+3 i\}$,

$\nu=\{\{1, m+1\} ;\{s(1), \ldots, s(m), 1\} ; 2 / 3+10 i / 3\}$. 
c. $\square$ indicates $R_{1 / 2}(\alpha)$, in scale.

indicates $R_{1 / 2}(\beta)$, in scale.

indicates $R_{1 / n}(\beta)$ (cannot be in scale).

indicates $R_{1 / n}(\gamma)$ (cannot be in scale).

indicates $R_{1 / n+1}(\delta)$ (cannot be in scale).

indicates $R_{1 / 2}(\zeta)$, in scale.

indicates $R_{1 / 2}(\theta)$, in scale.

$\square$ indicates $R_{1 / 3}(v)$, in scale.

Figure II illustrates a basic unit of construction (corresponding to the sequence $s$ ) of the only connected one of the "leaves." This "leaf" is $\left\{p \mid p_{11}=1\right\}$.

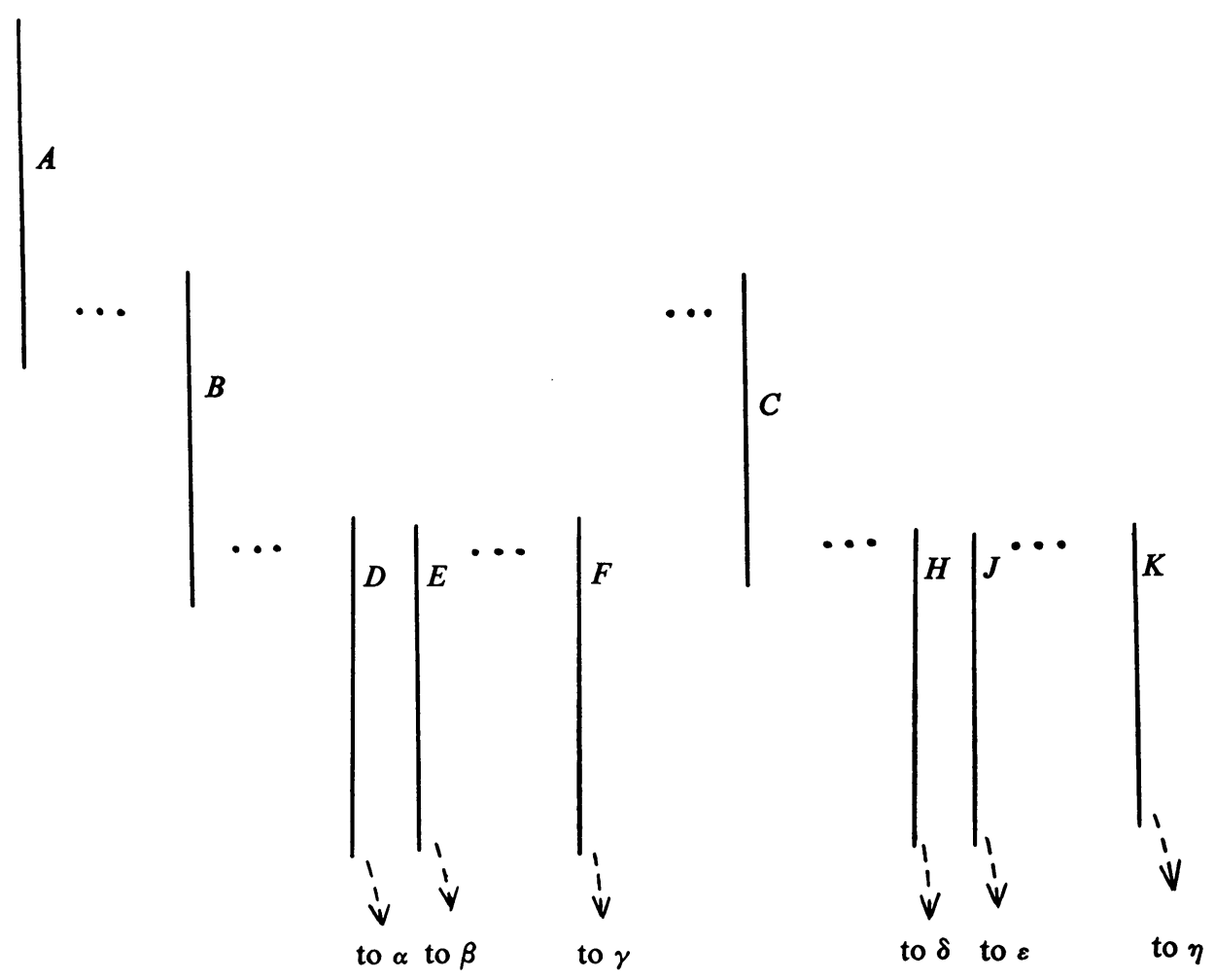

FIGURE III

\section{Legend for Figure III.}

a. III is a schematic drawing. The lines are supposed to represent rectangles as in Figures I and II. We suppose that each of $j, k, m$, and $n$ is a positive integer, $r$ is a number such that $0<r \leqq 1$, and $\{s(1)=1, s(2), \ldots, s(j)\}$ is a sequence of positive integers.

b. $A=\left\{p \mid p=\left\{\{r, j\} ;\{s\} ; p_{3}\right\}\right\}$,

$$
\begin{aligned}
& B=\left\{p \mid p=\left\{\{r, j+1\} ;\{s(1), \ldots, s(j), k\} ; p_{3}\right\}\right\}, \\
& C=\left\{p \mid p=\left\{\{r, j+1\} ;\{s(1), \ldots, s(j), 1\} ; p_{3}\right\}\right\}
\end{aligned}
$$




$$
\begin{aligned}
D & =\left\{p \mid p=\left\{\{r, j+2\} ;\{s(1), \ldots, s(j), k, m+1\} ; p_{3}\right\}\right\}, \\
E & =\left\{p \mid p=\left\{\{r, j+2\} ;\{s(1), \ldots, s(j), k, m\} ; p_{3}\right\}\right\}, \\
F & =\left\{p \mid p=\left\{\{r, j+2\} ;\{s(1), \ldots, s(j), k, 1\} ; p_{3}\right\}\right\}, \\
H & =\left\{p \mid p=\left\{\{r, j+2\} ;\{s(1), \ldots, s(j), 1, n+1\} ; p_{3}\right\}\right\}, \\
J & =\left\{p \mid p=\left\{\{r, j+2\} ;\{s(1), \ldots, s(j), 1, n\} ; p_{3}\right\}\right\}, \\
K & =\left\{p \mid p=\left\{\{r, j+2\} ;\{s(1), \ldots, s(j), 1,1\} ; p_{3}\right\}\right\}, \\
\text { c. } \alpha & =\left\{\{0, \infty\} ;\left\{\alpha_{2}(1)=s(1), \ldots, \alpha_{2}(j)=s(j), \alpha_{2}(j+1)=k, \alpha_{2}(j+2)=m+1, \ldots\right\}\right\}, \\
\beta & =\{\{0, \infty\} ;\{s(1), \ldots, s(j), k, m, \ldots\}\}, \\
\gamma & =\{\{0, \infty\} ;\{s(1), \ldots, s(j), k, 1, \ldots\}\}, \\
\delta & =\{\{0, \infty\} ;\{s(1), \ldots, s(j), 1, n+1, \ldots\}\}, \\
\varepsilon & =\{\{0, \infty\} ;\{s(1), \ldots, s(j), 1, n, \ldots\}\}, \\
\eta & =\{\{0, \infty\} ;\{s(1), \ldots, s(j), 1,1, \ldots\}\} .
\end{aligned}
$$

Figure III illustrates the relation between a "leaf," corresponding to some number $r$ in $(0,1]$, and the set $\left\{p \mid p_{11}=0\right\}$. This set plays the role of a "hinge" for the uncountably many "leaves," one for each number in $(0,1]$. The "leaves" corresponding to numbers in $(0,1)$ are copies of each other. The "leaf" corresponding to 1 is the only connected "leaf," and this leaf, together with the set $\left\{p \mid p_{11}=0\right\}$ connects up the various rectangles in the other "leaves."

2. Properties of the space. Some of the properties listed below can be proved in a straightforward manner and are indeed clear from the pictures given above.

(2.11) The space $\Gamma$ is a topological space.

(2.12) The space $\Gamma$ is regular.

(2.13) If $n$ is a positive integer, then $G_{n}$ covers $\Gamma$.

(2.14) If $p$ is a point in $\Gamma$ and $U$ is a region containing $p$ then there is a positive integer $n$ such that if each of $V$ and $W$ is in $G_{n}$ such that $p$ is in $V$ and $V \cdot W \neq 0$, then $V+W \subset U$.

(2.15) The space $\Gamma$ is metric. This follows from (2.12), (2.13), and (2.14), which states that $\Gamma$ satisfies the hypothesis of a metrization theorem due to R. L. Moore. This theorem can be found as the last theorem in [5].

(2.16) The space $\Gamma$ is complete $\left({ }^{14}\right)$. We modify the regions to the following extent: in (2.7), $\left|q_{3}-p_{3}\right|<\min \left\{1 / n, \operatorname{Im}\left(p_{3}\right) / 2\right\}$ for the first set and $\left|q_{3}-\left(p_{3}+3\right)\right|$ $<\min \left\{1 / n, \operatorname{Im}\left(p_{3}\right) / 2\right\}$ for the second set; and in (2.9)

$$
\left|q_{3}-p_{3}\right|<\min \left\{1 / n, 1-\operatorname{Im}\left(p_{3}\right), \operatorname{Im}\left(p_{3}\right) / 2\right\}
$$

for the first set and $\left|q_{3}-\left(p_{3}+2\right)\right|<\min \left\{1 / n, 1-\operatorname{Im}\left(p_{3}\right), \operatorname{Im}\left(p_{3}\right) / 2\right\}$ for the second

${ }^{(14)}$ A topological space is complete means that there is a monotone decreasing simple sequence $G$ of coverings (a covering is a collection of regions whose sum is the space) of $\Gamma$ such that (i) if $U$ is an open set containing a point $p$ then there is a positive integer $n$ so that every member of $G_{n}$, which contains $p$, lies in $U$, (such a $G$ is called a development for $\Gamma$ ), and (ii) if $C$ is a monotone decreasing sequence of closed sets so that $C_{n}$ is contained in some member of $G_{n}$ for each positive integer $n$, then $\prod_{n} C_{n} \neq 0$. 
set. Now defining $G^{\prime}$ in the same manner as $G$ but by using the new regions, we get a development of $\Gamma$ as needed to show completeness.

(2.17) If $p$ is a point with $p_{11}=0$, then $\Gamma$ is locally connected at $p$. Let $n$ be a positive integer and consider $R_{1 / n}(p)$. Let

$$
\begin{aligned}
X^{\prime} & =R_{1 / n}(p) \cdot\left\{q \mid q_{11}=1\right\} \\
& =\left\{q \mid q_{11}=1 ; q_{12} \geqq n ; q_{2}(j)=p_{2}(j) \text { for } j=1, \ldots, n\right\} .
\end{aligned}
$$

Let $X=X^{\prime} \cdot R_{1 / n}(p)$. Clearly, $X \supset X^{\prime}$ and $X \supset R_{1 / n}(p) \cdot\left\{q \mid q_{11}=0\right\}$. Also the set $X$ is connected because $X^{\prime}$ is connected. Now suppose that $R_{1 / n}(p)$ is not connected. Let $H$ and $K$ be two mutually separate $\left({ }^{15}\right)$ sets such that $H+K=R_{1 / n}(p)$. Suppose that $K \supset X$ and $u$ is a point in $H \cdot\left\{q \mid 0<q_{11}<1\right\}$. But $\left\{q \mid q_{1}=u_{1} ; q_{2}=u_{2}\right\}$ is connected and is contained in $R_{1 / n}(p)$, hence it is contained in $H$. Since $H$ is open, $H$ contains a point in each of all but at most finitely many of the sets $\left\{Y_{m} \mid m\right.$ is a positive integer; $Y_{m}=\left\{q \mid q_{11}=u_{11} ; q_{12}=u_{12}+1 ; q_{2}(j)=u_{2}(j)\right.$ for $j=1, \ldots, u_{12}$; $\left.q_{2}\left(q_{12}\right)=m\right\}$, and since each of these sets is connected, $H$ contains all but at most finitely many of these sets. Proceeding in this manner we conclude that there is a point $v$ with $v_{11}=0$ such that $v$ is a limit point of $H$. This contradicts our assumption that $R_{1 / n}(p)$ is not connected.

(2.18) The space $\Gamma$ is connected. This follows from (2.17) by observing that if $p_{11}=0$, then $R_{1}(p)=\Gamma$.

(2.19) If $p_{11} \neq 0$, then $\Gamma$ is locally compact $\left({ }^{16}\right)$ at $p$.

(2.20) The space $\Gamma$ is locally peripherally compact $\left({ }^{17}\right)$. For $p_{11} \neq 0$, this is obvious. Suppose that $p_{11}=0$. Let $n$ be a positive integer greater than 1 . The region $R_{1 / n}(p)$ has as its boundary

$$
\left\{q \mid q_{11}=1 ; q_{12}=n-1 ; q_{2}(j)=p_{2}(j) \text { for } j=1, \ldots, n-1 ; \operatorname{Im}\left(q_{3}\right)=1\right\},
$$

and this set is obviously compact.

(2.21) If $p_{11}=0$, then $\Gamma$ is locally peripherally connected at $p$. This follows from the fact that the boundary of $R_{1 / n}(p)$ in (2.20) is also connected.

(2.22) The space $\Gamma$ is locally remotely connected $\left({ }^{18}\right)$. The removal of sufficiently small regions from $\Gamma$ does not affect the argument in (2.17). So $\Gamma$ is also semilocally-connected.

(2.23) No countable set of points separates $\Gamma$. We observe that the set $H$ in (2.17)

${ }^{(15)}$ Two sets $H$ and $K$ are mutually separate means that neither contains a point nor a limit point of the other.

${ }^{\left({ }^{16}\right)}$ A set is compact means that every infinite subset has a limit point. Note that a compact set is not necessarily closed.

${ }^{\left({ }^{17}\right)}$ A space is locally peripherally compact at a point $p$ means that if $U$ is an open set containing $p$ then $U$ has an open subset $V$ which contains $p$ and whose boundary is compact.

${ }^{(18)}$ A space is locally remotely connected at a point $p$ means that if $U$ is an open set containing $p$ then $U$ has an open subset which contains $p$ and whose complement is connected. 
has uncountably many points in $\left\{p \mid p_{11}=0\right\}$ as its limit points. This observation, together with the structure of $\Gamma$ elsewhere, implies that the removal of a countable set will not affect the argument in (2.17).

(2.24) The set $\left\{p \mid p_{11}=0\right\}$ is separable. The set

$$
\left\{p \mid p_{11}=0 ; \text { for some positive integer } n, p_{2}(n)=p_{2}(m) \text { for } m \geqq n\right\}
$$

is a countable dense subset of $\left\{p \mid p_{11}=0\right\}$.

(2.25) If $p$ and $q$ are two points in $\Gamma$, then there is a separable continuum which contains both $p$ and $q$. Let

$$
X=\left\{v \mid v_{11}=p_{11}\right\}+\left\{v \mid v_{11}=q_{11}\right\}+\left\{v \mid v_{11}=0\right\}+\left\{v \mid v_{11}=1\right\} .
$$

By an argument similar to that in (2.17) we can show that $X$ is connected. Clearly $X$ is closed and separable. Hence, $X$ is a separable continuum containing $p$ and $q$.

(2.26) If $p$ and $q$ are points in $\Gamma$, then there is a separable continuum which separates $p$ from $q$. We shall discuss only the two difficult cases here.

Case 1. Suppose that $0<p_{11}=q_{11}<1, p_{2}=q_{2}$, and each of $\operatorname{Im}\left(p_{3}\right)$ and $\operatorname{Im}\left(q_{3}\right)$ is less than or equal to 1 , that is, we have the following:
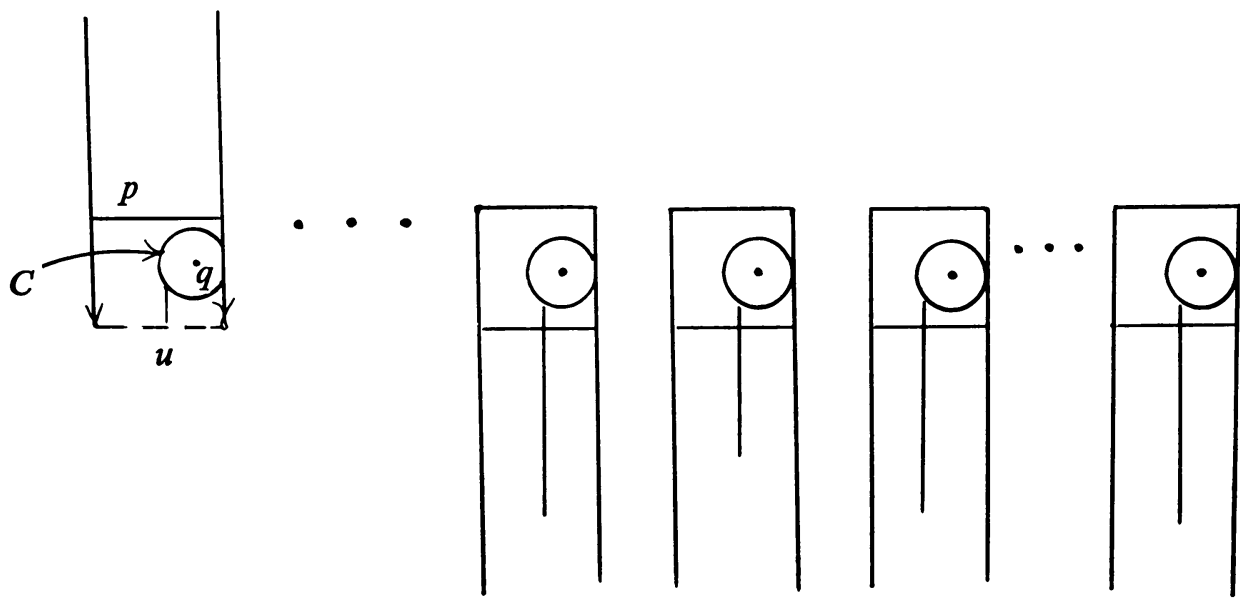

Let $C$ be a set as in the figure, namely, a circle or an arc of a circle, lying in the rectangle with height 2 , and separating $p$ from $q$ in that rectangle, and a vertical line segment, missing $p$ and $q$, intersecting the first set only at an end point, running to a point $u$ (not in $\Gamma$ ) at the bottom of the rectangle. We shall consider this point $u$ as a point in the complex plane, with the location of $u$ being determined by the imbedding in the complex plane of the rectangle in concern. Let

$$
\begin{gathered}
X=C+\left\{v \mid v_{11}=p_{11} ; v_{12}=p_{12}+1 ; v_{2}(j)=p_{2}(j) \text { for } j=1, \ldots, p_{12} ;\right. \\
\text { if } \left.\operatorname{Im}\left(v_{3}\right)>3, \text { then } v_{3} \text { is in } C+3 i, \text { if } \operatorname{Im}\left(v_{3}\right) \leqq 3, \text { then } \operatorname{Re}\left(v_{3}\right)=\operatorname{Re}(u)\right\} \\
+\left\{v \mid v_{11}=p_{11} ; v_{12}>p_{12}+1 ; v_{2}(j)=p_{2}(j) \text { for } j=1, \ldots, p_{12} ;\right. \\
\left.\operatorname{Re}\left(v_{3}\right)=\operatorname{Re}(u)\right\} .
\end{gathered}
$$


Let $Y=X+\left\{v \mid v_{11}=0\right.$ or $\left.v_{11}=1\right\}$. The closed set $Y$ can be shown to be connected by an argument similar to that in (2.17). $Y$ is separable because each of the sets whose sum is $Y$ is separable. So, $Y$ is a separable continuum which clearly separates $p$ from $q$.

Case 2. $p_{11}=q_{11}=1, p_{2}=q_{2}$, and each of $\operatorname{Im}\left(p_{3}\right)$ and $\operatorname{Im}\left(q_{3}\right)$ is less than or equal to 1 . In this case we have a situation similar to that in Case 1 , with the obvious differences. Define $C$ as in Case 1. If $C \cdot\left\{v \mid v_{1}=p_{1} ; v_{2}=p_{2} ; \operatorname{Im}\left(v_{3}\right)=1\right\} \neq 0$, let $C^{\prime}=C$. If $C \cdot\left\{v \mid v_{1}=p_{1} ; v_{2}=p_{2} ; \operatorname{Im}\left(v_{3}\right)=1\right\}=0$, let $C^{\prime}=C+\{$ a closed vertical line segment, missing $q$, intersecting $C$ only at one of its end points, and intersecting $\left\{v \mid v_{1}=p_{1} ; v_{2}=p_{2} ; \operatorname{Im}\left(v_{3}\right)=1\right\}$ only at its other end point $\}$. Define $X$ as in Case 1, replacing $C$ by $C^{\prime}$. Now $X$ is a separable continuum which clearly separates $p$ from $q$.

(2.27) The space $\Gamma$ is not separable.

Summarizing the properties of $\Gamma$, we have the following theorem:

THEOREM 2.1. There is a connected metric space $\Gamma$ such that

(1) $\Gamma$ is locally remotely connected,

(2) $\Gamma$ is locally peripherally compact,

(3) Every pair of points in $\Gamma$ belongs to a separable continuum,

(4) If $T=\{p \mid \Gamma$ is not locally compact at $p\}$ then $T$ is separable,

(5) $\Gamma$ is locally connected and locally peripherally connected at each point in $T$,

(6) Every pair of points in $\Gamma$ are separated by a separable continuum,

(7) No countable set of points separates $\Gamma$, but

(8) $\Gamma$ is not separable.

\section{Part III. Sets of Exceptional Points}

1. Sets exceptional with regard to local separability. In Theorem 1.1 it is assumed that the space is locally separable at each point. It is then natural to inquire for conditions on a subset $T$ of a connected metric space $S$ which would imply separability of $S$, provided that $S$ is locally separable at each point in $S-T$. It is highly plausible that separability of $T$ together with local peripheral separability of $S$ at each point in $T$ will be sufficient. That this is not the case is demonstrated by the space $\Gamma$. In fact, the space $\Gamma$ shows that even if we demand that $S$ be locally compact at each point in $S-T$ and locally peripherally compact at each point in $T$ and demand that $T$ be separable, we cannot conclude that $S$ is separable. One of the directions in which a solution seems possible is to require that the exceptional set $T$ be separable and behave like a point. This point-like behavior takes various forms both in this section and in the next.

If each of $p$ and $q$ is a point in a metric space then $d(p, q)$ denotes the distance between $p$ and $q$. If $p$ is a point in a metric space and $r$ is a positive number then $D_{r}(p)$ denotes the open disc with center $p$ and radius $r$. If $U$ is a subset of a metric space and the distances between pairs of points in $U$ is bounded, then the least 
such bound is called the diameter of $U$. If $C$ is a collection of sets then $C^{*}$ denotes the set which is the sum of all the sets in $C$.

LEMMA 3.1. If $U$ is an open subset of a connected metric space $S$ such that (i) $U \neq S$, (ii) $S$ is locally separable at each point in $S-U$, and (iii) $U$ is peripherally separable $\left({ }^{19}\right)$, then $S-U$ is separable.

Proof. For each point $p$ in $S-U$, let $\gamma(p)$ be the greatest number $\delta$ not greater than 1 such that $D_{\delta}(p)$ is separable. The set $D_{\gamma(p)}(p)$ is separable for each point $p$ in $S-U$. Let $B$ be the boundary of $U$ and let $C$ be a countable subcollection of $\left\{D_{\gamma(p)}(p) \mid p\right.$ in $\left.B\right\}$ such that $C$ covers $B$. This we can do by Theorem 1.6. Let $E_{1}$ be a countable dense subset of $C^{*}$, and for each positive integer $n$, let $E_{n+1}$ be a countable dense subset of $\sum_{p \in E_{n}} D_{\gamma(p)}(p)$. Let $F=(S-U) \cdot \sum_{n-1}^{\infty} E_{n}$. The set $F$ is countable and $B \subset \bar{F} \subset(S-U)$. Suppose that $\bar{F} \neq(S-U)$, that is $\bar{F}+U \neq S$. Let $q$ be a boundary point of the closed set $(\bar{F}+U)$. Since $U$ is open, $q$ is in $S-U$. Let $r$ be a point in $F$ such that $d(q, r)<\gamma(q) / 2$. It follows that $q \in D_{\gamma(q) / 2}(r) \subset D_{\gamma(q)}(q)$. Therefore $D_{\gamma(q) / 2}(r)$ is separable, which implies that $\gamma(q) / 2 \leqq \gamma(r)$. Hence $q \in D_{\gamma(r)}(r)$ $\subset(\bar{F}+U)$. Then $q$ cannot be a boundary point of $\bar{F}+U$. So $\bar{F}+U=S$, that is $\bar{F}=S-U$. Thus we have that $S-U$ is separable.

DEFINITION 3.1. A metric space $S$ is locally peripherally separable about a subset $T$ means that there is a simple sequence $\left\{U_{1} \supset U_{2} \supset U_{3} \cdots\right\}$ of peripherally separable open sets such that if $n$ is a positive integer, then $T \subset U_{n} \subset \sum_{t \in T} D_{1 / n}(t)$.

THEOREM 3.1. If $T$ is a subset of a connected metric space $S$ such that (i) $S$ is locally separable at each point in $S-T$, (ii) $S$ is locally peripherally separable about $T$, and (iii) $T$ is separable, then the space $S$ is separable.

Proof. We assume that $T$ is not dense in $S$, because otherwise $S$ is obviously separable. Let $\left\{U_{1} \supset U_{2} \supset U_{3} \supset \cdots\right\}$ be a simple sequence of peripherally separable open sets such that if $n$ is a positive integer, then $U_{n} \neq S$ and $T \subset U_{n} \subset \sum_{t \in T} D_{1 / n}(t)$. Now we observe that

$$
S=\sum_{n=1}^{\infty}\left(S-U_{n}\right)+\prod_{n=1}^{\infty} U_{n} \text { and } \prod_{n=1}^{\infty} U_{n} \subset \bar{T} .
$$

By Lemma 3.1, $S-U_{n}$ is separable for each positive integer $n$. Because $T$ is separable, $\bar{T}$ is separable, and hence, $\prod_{n=1}^{\infty} U_{n}$ is separable. Since the sum of countably many separable sets is separable, we have that $S$ is separable.

The hypothesis of Theorem 3.1 involves the behavior of $S$ with respect to the set $T$ as a whole. We now give a condition which deals with only the behavior of $S$ at each point in $T$.

Definition 3.2( $\left.{ }^{20}\right)$. A subset $T$ of a metric space $S$ has property $A$ means that if

(19) A set is peripherally separable means that the set has a separable boundary.

$\left({ }^{20}\right)$ See $\S 3$ of this part for a discussion of property A. 
$C$ is an open covering of $T$ such that $C$ contains a local basis $\left({ }^{21}\right)$ for each point in $T$, then $C$ has a subcollection $E$ which covers $T$ and if $n$ is a positive integer then at most finitely many members of $E$ have diameters greater than $1 / n$.

THEOREM 3.2. If $T$ is a subset of a connected metric space $S$ such that (i) $S$ is locally separable at each point in $S-T$, (ii) $S$ is locally peripherally separable at each point in $T$, and (iii) $T$ has property $\mathrm{A}$, then $S$ is separable.

Proof. The set $T$ is clearly separable. We assume that $T$ is not dense in $S$, because otherwise $S$ is obviously separable. Let $M$ be a positive integer such that

$$
\sum_{t \in T} D_{1 / M}(t) \neq S
$$

Now we let

$X_{1}=$ a collection to which $Z$ belongs only in case $Z$ is a peripherally separable open set and there is a point $t$ in $T$ such that $t \in Z \subset D_{1 / M}(t),\left(X_{1}\right.$ covers $T$ and contains a local basis for each point in $T$ ),

$Y_{1}=$ a countable subcollection of $X_{1}$ such that $Y_{1}$ covers $T$ and if $m$ is a positive integer then at most finitely many members of $Y_{1}$ have diameters greater than $1 / \mathrm{m}$, and $U_{1}=Y_{1}^{*}$. For each positive integer $n$, let

$X_{n+1}=$ a collection to which $Z$ belongs only in case $Z$ is a peripherally separable open set and there is a point $t$ in $T$ such that $t \in Z \subset D_{1 / M+n}(t) \cdot U_{n},\left(X_{n+1}\right.$ covers $T$ and contains a local basis for each point in $T$ ),

$Y_{n+1}=$ a countable subcollection of $X_{n+1}$ such that $Y_{n+1}$ covers $T$ and if $m$ is a positive integer then at most finitely many members of

$Y_{n+1}$ have diameters greater than $1 / m$, and $U_{n+1}=Y_{n+1}^{*}$.

Suppose that $N$ is a positive integer. Clearly, $U_{N} \neq S$. Let $B$ be the boundary of $U_{N}$ and let $C$ be the sum of the boundaries of members of $Y_{N}$. Since $Y_{N}$ is a countable collection and the boundary of each member of $Y_{N}$ is separable, $C$ is separable. If $B$ is a subset of $C$ then $B$ is obviously separable. Suppose that $B$ is not a subset of $C$. Let $q$ be a point in $B$ such that $q$ is not in $C$. We shall show that $q$ is a limit point of $C$. Let $j$ be a positive integer. There are at most finitely many members of $Y_{N}$ with diameters greater than $1 / 2 j$. Since $q$ is not in $C$, we can let $k$ be a positive integer with $1 / k<1 / 4 j$ such that if $Z$ is a member of $Y_{N}$ with diameter greater than $1 / 2 j$, then $D_{1 / k}(q) \cdot Z=0$. Let $r$ be a point in $U_{N}$ such that $d(q, r)<1 / k$, and let $W$ be a member of $Y_{N}$ such that $r$ belongs to $W$. The diameter of $W$ is less than or equal to $1 / 2 j$. Therefore, $W \subset D_{3 / 4 j}(q)$, and $\bar{W} \subset D_{1 / j}(q)$. Hence, $D_{1 / j}(q)$ contains a point of $C$. This proves that $q$ is a limit point of $C$. Now we can conclude that $B \subset \bar{C}$, which means that $B$ is separable.

$\left({ }^{21}\right)$ If $S$ is a topological space and $p$ is a point in $S$, then $G$ is a local basis for $p$ means that $G$ is a collection of open sets containing $p$ such that every open set containing $p$ has as a subset a member of $G$. 
Thus, for each positive integer $n, U_{n}$ is an open set with a separable boundary, and $T \subset U_{n} \subset \sum_{t \in T} D_{1 / n}(t)$, that is, $S$ is locally peripherally separable about $T$. It follows then, by Theorem 3.1, that $S$ is separable.

The next theorem is an interesting special case of Theorem 3.2.

THEOREM 3.3. If $T$ is a subset of a connected metric space $S$ such that (i) $S$ is locally separable at each point in $S-T$ (ii) $S$ is locally peripherally separable at each point in $T$, and (iii) $T$ is the sum of countably many compact sets, then $S$ is separable.

Proof. Let $\left\{C_{1}, C_{2}, C_{3}, \ldots\right\}$ be a sequence of compact sets such that $T=\sum_{n=1}^{\infty} C_{n}$. Let $T^{\prime}=\sum_{n=1}^{\infty} \bar{C}_{n}$. Suppose that $E$ is an open covering of $T^{\prime}$ such that $E$ contains a local basis for each point in $T^{\prime}$. For each positive integer $n$ let $F_{n}$ be a finite subcollection of $\{H \mid H \in E$ and the diameter of $H$ is less than $1 / n\}$ such that $F_{n}$ covers $\bar{C}_{n}$ (this can be done because $\bar{C}_{n}$ is a closed and compact set). It follows that $\sum_{n=1}^{\infty} F_{n}$ is a subcollection of $E$ such that if $m$ is a positive integer, at most finitely many members of $\sum_{n=1}^{\infty} F_{n}$ have diameters greater than $1 / m$. Thus $T^{\prime}$ has property A. Now, by Theorem 3.2, $S$ is separable.

A corollary to Theorem 3.3 is the following theorem.

THEOREM 3.4 (TREYBIG [11]). If $T$ is a subset of a connected metric space $S$ such that (i) $S$ is locally separable at each point in $S-T$, (ii) $S$ is locally peripherally separable at each point in $T$, and (iii) $T$ is countable, then $S$ is separable.

The conditions on the exceptional set $T$ in the above theorems involved separability conditions on the space $S$ near the set $T$. We now give a similar condition on $T$ where the separability conditions on $S$ near $T$ are replaced by a connectedness property of $S$ near $T$.

Definition 3.3. A metric space $S$ is locally peripherally sigma-connected about a subset $T$ means that there is a simple sequence $\left\{U_{1} \supset U_{2} \supset U_{3} \supset \ldots\right\}$ of peripherally sigma-connected $\left({ }^{22}\right)$ open sets such that if $n$ is a positive integer, then

$$
T \subset U_{n} \subset \sum_{t \in T} D_{1 / n}(t) .
$$

THEOREM 3.5. If $T$ is a subset of a connected metric space $S$ such that (i) $S$ is locally separable at each point in $S-T$, (ii) $S$ is locally peripherally sigma-connected about $T$, and (iii) $T$ is separable, then $S$ is separable.

Proof. Let $\left\{U_{1} \supset U_{2} \supset U_{3} \supset \cdots\right\}$ be a sequence as described in Definition 3.3. Let $n$ be a positive integer, $B$ the boundary of $U_{n}$, and $C$ be the countable set of connected sets such that $B=C^{*}$. Let $E$ be a member of $C$. Since $E \subset S-T$, we have that $S$ is locally separable at each point in $E$. Hence, by Theorems 1.1 and 1.5 , the set $E$ is separable. Thus, each member of $C$ is separable. It follows that, since $C$ is

$\left.{ }^{(22}\right)$ A set is peripherally sigma-connected means that the boundary of the set is the sum of at most countably many connected sets. 
countable, $C^{*}=B$ is separable. Therefore $U_{m}$ is peripherally separable for each positive integer $m$, that is, $S$ is locally peripherally separable about $T$. Now, by Theorem 3.1, we have that $S$ is separable.

2. Sets exceptional with regard to local connectedness. The problem suggested by Theorem 1.1 and studied in the preceding section has an analogue which is suggested by Theorem 1.2. Here we seek conditions on a subset $T$ of a connected metric space $S$ which would imply the separability of $S$, provided that $S$ is locally connected and locally peripherally separable at each point in $S-T$. In this case the answer is more direct than in the previous situation.

LEMMA 3.2. If $U$ is an open subset of a connected metric space $S$ such that (i) $U \neq S$, (ii) $S$ is locally connected and locally peripherally separable at each point in $S-U$, and (iii) $U$ is peripherally separable, then $S-U$ is separable.

Proof. For each point $p$ in $S-U$ and each positive integer $n$, let $\gamma(p, n)$ be the least positive integer $\delta$ for which there is a peripherally separable open set $V$ such that $D_{1 / \delta}(p) \subset V \subset D_{1 / n}(p)$, and let $V(p, n)$ be a peripherally separable open set such that $D_{1 / \gamma(p, n)}(p) \subset V(p, n) \subset D_{1 / n}(p)$. Let $B$ be the boundary of $U$, and let $C$ be a countable dense subset of $B$. Let $E_{1}$ be a countable dense subset of

$$
\sum_{p \in C} \sum_{n=1}^{\infty}\{\text { Boundary of } V(p, n)\},
$$

and if $m$ is a positive integer, let $E_{m+1}$ be a countable dense subset of

$$
\sum_{p \in E_{m}} \sum_{n=1}^{\infty}\{\text { Boundary of } V(p, n)\} \text {. }
$$

Let $F=(S-U) \cdot \sum_{n=1}^{\infty} E_{n}$. Clearly, $F$ is countable and $B \subset \bar{F} \subset(S-U)$. Hence $\bar{F}+U$ is a closed set. Assume that $\bar{F} \neq S-U$, that is, $S \neq \bar{F}+U$. The set $S-(\bar{F}+U)$ is an open set, and each component of $S-(\bar{F}+U)$ is an open set, because $S$ is locally connected at each point in $S-U$. Let $H$ be a component of $S-(\bar{F}+U)$, and let $q$ be a boundary point of $H$. The point $q$ belongs to $\bar{F}$. Let $j$ be a positive integer such that $D_{1 / j}(q)$ does not contain $H$. Let $r$ be a point in $F$ such that $d(q, r)<1 / 2 \gamma(q, 4 j)$. We then have the following relations:

$$
q+r \subset D_{1 / 2 \gamma(q, 4 j)}(r) \subset D_{1 / \gamma(q, 4 j)}(q) \subset V(q, 4 j) \subset D_{1 / 4 j}(q) \subset D_{1 / 2 j}(r) \subset D_{1 / j}(q) .
$$

It then follows from the definition of $\gamma(p, n)$ that $\gamma(r, 2 j) \leqq 2 \gamma(q, 4 j)$, which implies, by the definition of $V(p, n)$, that $q$ belongs to $V(r, 2 j)$. Also from the above relations it is clear that $V(r, 2 j)$ does not contain $H$. So, we have that the connected set $H$ intersects both the open set $V(r, 2 j)$ and its complement. Hence, $H$ contains a boundary point of $V(r, 2 j)$. But every boundary point of $V(r, 2 j)$ belongs to $\bar{F}$. So, $H$ contains a point of $\bar{F}$ and, hence, $H$ cannot be a subset of $S-(\bar{F}+U)$. Therefore, $S=\bar{F}+U$, that is, $\bar{F}=S-U$. Thus, we have that $S-U$ is separable.

THEOREM 3.6. If $T$ is a subset of a connected metric space $S$ such that (i) $S$ is locally connected and locally peripherally separable at each point in $S-T$, (ii) $S$ is locally peripherally separable at each point in $T$, and (iii) $T$ is separable, then $S$ is separable. 
Proof. We assume that $T$ is not dense in $S$, because otherwise $S$ is obviously separable. Let $M$ be a positive integer such that $S \neq \sum_{t \in T} D_{1 / M}(t)$. Let

$X_{1}=$ a collection to which $Z$ belongs only in case $Z$ is a peripherally separable open set and there is a point $t$ in $T$ such that $t \in Z \subset D_{1 / M}(t)$, (clearly, $X_{1}$ is an open covering of $T$ ),

$Y_{1}=$ a countable subcollection of $X_{1}$ such that $Y_{1}$ covers $T$, and $U_{1}=Y_{1}^{*}$. If $n$ is a positive integer, let

$X_{n+1}=$ a collection to which $A$ belongs only in case $Z$ is a peripherally separable open set and there is a point $t$ in $T$ such that $t \in Z \subset U_{n} \cdot D_{1 / M+n}(t)$, (clearly, $X_{n+1}$ is an open covering of $T$ ),

$Y_{n+1}=$ a countable subcollection of $X_{n+1}$ such that $Y_{n+1}$ covers $T$, and $U_{n+1}=Y_{n+1}^{*}$. Now we observe that

$$
S=\sum_{n=1}^{\infty}\left(S-U_{n}\right)+\prod_{n=1}^{\infty} U_{n} \text { and } \prod_{n=1}^{\infty} U_{n} \subset \bar{T} .
$$

Since $\bar{T}$ is separable, $\prod_{n=1}^{\infty} U_{n}$ is separable. We shall now show that $S-U_{n}$ is separable for each positive integer $n$. Suppose that $N$ is a positive integer. Since $U_{N} \neq S$, we can let $B$ be the boundary of $U_{N}$, and let $C$ be the sum of the boundaries of members of $Y_{N}$. Since each member of $Y_{N}$ is peripherally separable and $Y_{N}$ is a countable collection, $C$ is separable. If $B$ is a subset of $C$, then $B$ is clearly separable. Assume that $B$ is not a subset of $C$ and let $q$ be a point in $B$ which is not in $C$. We shall show that $q$ is a limit point of $C$. Let $j$ be a positive integer. Because $S$ is locally connected at each point in $B$, we can let $E$ be a connected open set such that $q \in E \subset D_{1 / j}(q)$. Let $r$ be a point of $U_{N}$ in $E$ and let $W$ be a member of $Y_{N}$ such that $W$ contains $r$. Now $E$ is a connected set which contains the point $r$ in the open set $W$ and which contains the point $q$ in the complement of $W$. Hence, $E$ contains a point in the boundary of $W$, that is, $D_{1 / j}(q)$ contains a point in $C$. Therefore, $q$ is a limit point of $C$. This shows that $B \subset \bar{C}$, which implies that $B$, the boundary of $U_{N}$, is separable. Therefore, by Lemma $3.2, S-U_{N}$ is separable. Hence, $S-U_{n}$ is separable for each positive integer $n$. Thus, we have that the space $S$ is separable.

The next theorem gives an apparent weakening of condition (i) in Theorem 3.6.

THEOREM 3.7. If $T$ is a subset of a connected metric space $S$ such that (i) $S$ is connected im kleinen $\left({ }^{23}\right)$ and locally peripherally separable at each point in $S-T$, (ii) $S$ is locally peripherally separable at each point in $T$, and (iii) $T$ is separable, then $S$ is separable.

Proof. Consider the case where $\bar{T} \neq S$, because otherwise it is obvious that $S$ is separable. If a space is connected im kleinen at each point of an open set $U$ then

$\left({ }^{23}\right)$ A topological space $S$ is connected im kleinen at a point $p$ means that if $U$ is an open set containing $p$ then there is a continuum $C$ and an open set $V$ such that $p \in V \subset C \subset U$. Note that a space may be connected im kleinen at a point $p$ without being locally connected at $p$. 
it is locally connected at each point of $U\left({ }^{24}\right)$. Thus $S$ is locally connected at each point in $S-\bar{T}$. Now, by Theorem 3.6, with $\bar{T}$ as the set of exceptional points, we have that $S$ is separable.

The following theorem is another apparent weakening of condition (i) of Theorem 3.6. It is also a solution to a version of the main problem in this part, suggested by Theorem 1.3 and concerning sets exceptional with regard to locally peripheral connectedness.

THEOREM 3.8. If $T$ is a subset of a connected metric space $S$ such that (i) $S$ is locally peripherally connected and locally peripherally separable at each point in $S-T$, (ii) $S$ is locally peripherally separable at each point in $T$, and (iii) $T$ is separable, then $S$ is separable.

Proof. Consider the case where $\bar{T} \neq S$, for otherwise it is clear that $S$ is separable. If a space is locally peripherally connected at each point of an open set $U$ then it is locally connected at each point in $U\left({ }^{25}\right)$. Thus $S$ is locally connected at each point in $S-\bar{T}$. Now, by Theorem 3.6, with $\bar{T}$ as the set of exceptional points, we have that $S$ is separable.

Now, exactly as in the preceding section, we replace the separability behavior of $S$ near $T$ by a connectedness property of $S$ near $T$.

THEOREM 3.9. If $T$ is a closed subset of a connected metric space $S$ such that (i) $S$ is locally connected and locally peripherally separable at each point in $S-T$, (ii) $S$ is locally peripherally sigma-connected about $T$, and (iii) $T$ is separable, then $S$ is separable.

Proof. Let $\left\{U_{1} \supset U_{2} \supset U_{3} \supset \cdots\right\}$ be a sequence as described in Definition 3.3. Let $n$ be a positive integer, $B$ be the boundary of $U_{n}$. B has at most countably many components. Let $C$ be a component of $B$. For each point $p$ in $C$, let $V_{p}$ be a connected open set containing $p$ such that $T \cdot V_{p}=0$. Since $V_{p}+C$ is connected for each $p$ in $C$, we have that $W=\sum_{p \in C} V_{p}$ is a connected open set such that $C \subset W$. The relative topology of $W$ has the properties that $W$ is locally connected and locally peripherally separable at each point in $W$. Hence, by Theorem 1.2, W is separable. Then, by Theorem 1.5, $C$ is separable. Hence each of the countable components of $B$ is separable. This implies that $B$ is separable. Thus $S$ is locally peripherally separable about $T$. Now, as in Theorem 3.6, we can show that $S$ is separable.

3. Remarks and examples. As shown in Theorem 3.3, the sum of countably many closed and compact sets has property A. Clearly every set which has property A is separable. But there are separable sets which do not have property A. For example, in view of Theorem 3.2 and Theorem 2.1 , the set, denoted by $\left\{p \mid p_{11}=0\right\}$ in the space $\Gamma$ described in Chapter II, is separable but does not have property A.

(24) This is a well-known result.

$\left({ }^{25}\right)$ This is a well-known result. 
The sharpness of the results in this part may be seen by the statement of Theorem 2.1. For instance, Theorems 1.1 and 1.2 suggest strongly that if ach point in a connected metric space, the space is either (i) locally separable, or (ii) locally peripherally separable and locally connected, then the space is separable. Theorem 2.1 shows that this is not the case.

Theorem 3.2 seems to suggest that, in Theorem 3.5, one can replace the condition (ii) by (ii)' $S$ is locally connected at each point in $T$, or by (ii)" $S$ is locally peripherally connected at each point of $T$, and condition (iii) by (iii)' $T$ has property A. That this is not the case can be seen by the following example:

Let $S$ be a space made up of uncountably many copies of the unit square: $\{z \mid 0 \leqq \operatorname{Re}(z) \leqq 1$, and $0 \leqq \operatorname{Im}(z) \leqq 1\}$, attached to each other along the interval $\{z \mid 0 \leqq \operatorname{Re}(z) \leqq 1$; and $\operatorname{Im}(z)=0\}$. Suppose that $p$ and $q$ are two points in $S$. If $p$ and $q$ are in the same square, the distance between $p$ and $q$ is $|p-q|$. If $p$ and $q$ are in different squares, the distance between $p$ and $q$ is $p^{*}-q$.

The above space also serves as a counterexample to the proposition that the condition (ii) in Theorem 3.9 can be replaced by the condition (ii)' or by (ii)" above.

For Theorem 3.9, the words "locally connected" in condition (i) can be replaced by either "connected im kleinen" or "locally peripherally connected." The alterations in the proof of Theorem 3.9 required are exactly those in the proof of Theorem 3.6 as given for Theorems 3.7 and 3.8.

\section{Part IV. Some General Conditions For Separability}

1. The general situation. In this section we give some conditions for the separability of a metric space where it is not assumed that the space is locally connected. In a space $S$, a subset $T$ is said to separate two subsets $U$ and $V$ provided that there are mutually separate subsets $H$ and $K$ such that $S-T=H+K, U \subset H$, and $V \subset K$.

LEMMA 4.1. If $T$ is a subset with property $\mathrm{A}$ of a metric space $S$ and $E$ is a subset of $T$ such that $\bar{E} \cdot T=E$, (that is, $E$ is closed with respect to the relative topology of $T)$, then $E$ has property A.

Proof. Suppose that $G$ is an open covering of $E$ such that $G$ contains a local basis for each point in $E$. For each point $p$ in $T-E$, let $H_{p}$ be an open set such that $p \in H_{p} \subset S-E$. This can be done because no point in $T-E$ is a limit point of $E$. Let $J$ be the collection of open sets to which $Z$ belongs only in case $Z$ is an open subset of $H_{p}$ for some $p$ in $T-E$. Clearly $G+J$ is an open covering of $T$ which contains a local basis for each point in $T$. Let $K$ be a subcollection of $G+J$ such that $K$ covers $T$ and if $m$ is a positive integer then at most finitely many members of $K$ have diameters greater than $1 / \mathrm{m}$. Since no member of $J$ contains a 
point in $E, G \cdot K$ covers $E$ and has the desired property that at most finitely many members of $G \cdot K$ have diameters greater than a given positive number. Hence, $E$ has property $\mathrm{A}$.

LEMMA 4.2. If $S$ is a metric space such that for every two open subsets of $S$, there is a connected set with property A which intersects both the open sets, then every set with property $\mathrm{A}$ is contained in a connected set with property $\mathrm{A}$.

Proof. For each two points $p$ and $q$ and each positive integer $n$, let $\Delta(p, q, n)$ be a connected set with property A which intersects both $D_{1 / n}(p)$ and $D_{1 / n}(q)$. Suppose that $T$ is a subset with property A of $S$. Let $E_{1}=$ a countable dense subset of $T$, and $F_{1}=T+\sum_{p, q \in E_{1}} \sum_{n=1}^{\infty} \Delta(p, q, n)$, and if $m$ is a positive integer, let $E_{m+1}=$ a countable dense subset of $F_{m}$, and $F_{m+1}=F_{m}+\sum_{p, q \in E_{m+1}} \sum_{n=1}^{\infty} \Delta(p, q, n)$. Let $G=\sum_{m=1}^{\infty} F_{m}$. Since $G$ is the sum of countably many sets with property A, $G$ has property A. Also $J=\sum_{m=1}^{\infty} E_{m}$ is a countable set which is dense in $G$. We shall show that $G$ is connected.

Suppose, on the contrary, that $G$ is not connected. Let $G=H+K$, where $H$ and $K$ are two mutually separate sets. Let $p$ be a point in $H$ and $q$ be a point in $K$. Let $N$ be a positive integer such that $H \cdot D_{1 / N}(q)=0$ and $K \cdot D_{1 / N}(p)=0$. Now we can let $p^{\prime}$ and $q^{\prime}$ be points in $J$ such that $d\left(p, p^{\prime}\right)<1 / 2 N$, and $d\left(q, q^{\prime}\right)<1 / 2 N$. It follows that $\Delta\left(p^{\prime}, q^{\prime}, 2 N\right)$ is a connected subset of $G$, and hence is contained in either $H$ or $K$. But this contradicts the fact that $\Delta\left(p^{\prime}, q^{\prime}, 2 N\right)$ intersects both $D_{1 / N}(p)$ and $D_{1 / N}(q)$. Hence, $G$ is a connected set with property A and $T \subset G$.

THEOREM 4.1. If $S$ is a connected metric space such that (i) $S$ is locally peripherally separable, and (ii) for every two open sets, there is a connected set with property A which intersects both the open sets, then the space $S$ is separable.

Proof. For each point $p$ in $S$ and each positive integer $n$, let $\gamma(p, n)$ be the least positive integer $\delta$ for which there is a peripherally separable open set $V$ such that $D_{1 / \delta}(p) \subset V \subset D_{1 / n}(p)$, and let $V(p, n)$ be a peripherally separable open set such that $D_{1 / \gamma(p, n)}(p) \subset V(p, n) \subset D_{1 / n}(p)$. Let $\sigma$ be a point in $S$ and let $X_{1}=$ a countable dense subset of $\sum_{n=1}^{\infty}$ \{Boundary of $\left.V(\sigma, n)\right\}$, and if $m$ is a positive integer, let $X_{m+1}=\mathrm{a}$ countable dense subset of $\sum_{p \in X_{m}} \sum_{n=1}^{\infty}$ \{Boundary of $\left.V(p, n)\right\}$. Let $Y=\sum_{m=1}^{\infty} X_{m}$. Clearly, $\bar{Y}$ is separable and $\sum_{p \in Y} \sum_{m=1}^{\infty}$ \{Boundary of $\left.V(p, n)\right\}$ is a subset of $\bar{Y}$. We shall show that $\bar{Y}=S$.

Suppose, on the contrary, that $\bar{Y} \neq S$. Let $r$ be a point in the open set $S-\bar{Y}$, $B$ be the boundary of $S-\bar{Y}, j$ be a positive integer such that $D_{1 / j}(r) \cdot B=0$, and $t$ be a point in $B$. By Lemma 4.2, we can let $C$ be a connected set with property A such that $C$ contains $r$ and $t$. Let $E=B \cdot C$. Since $B$ is closed $\bar{E} \subset B$. Hence $\bar{E} \cdot C=E$. Therefore, by Lemma $4.1, E$ has property A. Suppose that $p$ is a point in $E$ and $k$ is a positive integer greater than $2 j$. Let $q$ be a point in $Y$ such that $d(p, q)<$ $1 / 2 \gamma(p, 4 k)$. Now we have

$p+q \subset D_{1 / 2 \gamma(p, 4 k)}(q) \subset D_{1 / \gamma(p, 4 k)}(p) \subset V(p, 4 k) \subset D_{1 / 4 k}(p) \subset D_{1 / 2 k}(q) \subset D_{1 / k}(p)$. 
From the definition of $\gamma$ it follows that $\gamma(q, 2 k) \leqq 2 \gamma(p, 4 k)$. Consequently, $p \in V(q, 2 k) \subset D_{1 / k}(p)$. Therefore, we can choose a subcollection $F$ of

$$
\{V(q, n) \mid q \in Y \text { and } n=\text { a positive integer }\}
$$

such that $F$ covers $E, F$ contains a local basis for each point in $E$, and $F^{*} \subset$ $\sum_{p \in E} D_{1 / 2 j}(p)$. Hence, $r \in S-\bar{F}^{*}$. Since $E$ has property A, we can let $H$ be a subcollection of $F$ such that $H$ covers $E$ and at most finitely many members of $H$ have diameters greater than a given positive number. Let $J$ be the boundary of $H^{*}$ and $K$ be the sum of the boundaries of members of $H$. The argument in the second paragraph of the proof of Theorem 3.2 shows that $J \subset \bar{K} \subset \bar{Y}$. Let $L=(S-\bar{Y})-\bar{H}^{*}$. Because the connected set $C$ contains the point $r$ in the open set $L$ and the point $t$ in the complement of $L$, we can let $u$ be a point of the boundary of $L$ such that $u$ is in $C$. The point $u$ belongs to $\bar{Y}+\bar{H}^{*}$. But $u$ is not in $B$, because every point in $E=B \cdot C$ belongs to the open set $H^{*}$. Consequently, $u$ is not in $\bar{Y}$ and hence $u$ is in $\bar{H}^{*}$. Then $u$ is in $J$ and therefore $u$ is in $\bar{Y}$. This is a contradiction. So we must have that $S=\bar{Y}$. Thus, we have that $S$ is separable.

As a corollary to Theorem 4.1, we get the following criterion for separability.

TheOREM 4.2 (TREYBIG [7]). If $S$ is a connected metric space such that (i) $S$ is locally peripherally separable and (ii) $S$ is compact-continuum-wise connected $\left({ }^{26}\right)$, then $S$ is separable.

However, the above theorem of Treybig suggests the following condition for separability.

THEOREM 4.3. If $S$ is a connected metric space such that (i) every two points $p$ and $q$ in $S$ are separated by some separable closed set and (ii) for any two points $p$ and $q$ and any open set, there is a compact continuum which lies in $S-q$, contains $p$, and intersects the open set, then the space $S$ is separable.

Proof. For each two points $p$ and $q$ in $S$, let $\gamma(p, q)$ be the least positive integer $\delta$ such that there is a separable closed set which separates $D_{1 / \delta}(p)$ from $D_{1 / \delta}(q)$, and let $V(p, q)$ be a separable closed set which separates $D_{1 / \gamma(p, q)}(p)$ from $D_{1 / \gamma(p, q)}(q)$. Let $p_{0}$ and $q_{0}$ be two points in $S$, and let

$X_{0}=$ a countable dense subset of $V\left(p_{0}, q_{0}\right)$, and if $m$ is a nonnegative integer, then let

$X_{m+1}=$ a countable dense subset of $\left\{\sum_{p \neq q \in X_{m}} V(p, q)+X_{m}\right\}$. Let $Y=\sum_{m=0}^{\infty} X_{m}$. Clearly, $Y$ is countable. We shall show that $S=\bar{Y}$.

Suppose that, on the contrary, $S \neq \bar{Y}$. Let $r$ be a point in the open set $S-\bar{Y}, t$ be a point in $\bar{Y}$, and $j$ be a positive integer such that $D_{1 / j}(r) \cdot \bar{Y}=0$. For each positive integer $n$, let $C_{n}$ be a compact continuum containing $r$ and intersecting $D_{1 / 2 j+n}(t)$.

$\left({ }^{26}\right)$ A space is compact-continuum-wise connected means that every two points in the space belong to a compact continuum. 
If there is a positive integer $n$ such that $\bar{Y} \cdot C_{n} \neq 0$; then let $N$ be such a positive integer, $E$ be a component of $C_{N} \cdot(S-\bar{Y})$ such that $E$ contains $r$, and $u$ be a boundary point $\left({ }^{27}\right)$ of $S-\bar{Y}$ such that $u$ is in $\bar{E}$. If $\bar{Y} \cdot C_{n}=0$ for each positive integer $n$, then let $u=t$. Let $w$ be a point in $\bar{Y}$ such that $u \neq w$, and for each positive integer $n$, let $F_{n}$ be a compact continuum containing $r$ and intersecting $D_{1 / 2 j+n}(w)$. If there is a positive integer $n$ such that $\bar{Y} \cdot F_{n} \neq 0$; then let $M$ be such a positive integer, $J$ be the component of $F_{M} \cdot(S-\bar{Y})$ such that $J$ contains $r$, and $z$ be a boundary point of $S-\bar{Y}$ such that $z$ is in $\bar{J}$. If $\bar{Y} \cdot F_{n}=0$ for each positive integer $n$, then let $z=w$. It follows that for any positive integer $n$, there is a connected set lying in $S-\bar{Y}$ which intersects both $D_{1 / n}(u)$ and $D_{1 / n}(z)$. Let $\sigma$ and $\pi$ be points in $Y$ such that $\sigma \in D_{1 / 2 \gamma(u, z)}(u)$ and $\pi \in D_{1 / 2 \gamma(u, z)}(z)$, and let $Q$ be a positive integer such that $X_{Q}$ contains both $\sigma$ and $\pi$. It follows that $u \in D_{1 / 2 \gamma(u, z)}(\sigma) \subset D_{1 / \gamma(u, z)}(u)$ and $z \in D_{1 / 2 \gamma(u, z)}(\pi) \subset D_{1 / \gamma(u, z)}(z)$. Hence, $V(u, z)$ separates $D_{1 / 2 \gamma(u, z)}(\sigma)$ from $D_{1 / 2 \gamma(u, z)}(\pi)$. Then, by definition, $\gamma(\sigma, \pi) \leqq 2 \gamma(u, z)$. Consequently, $V(\sigma, \pi)$ separates $D_{1 / 2 \gamma(u, z)}(\sigma)$ from $D_{1 / 2 \gamma(u, z)}(\pi)$, and hence $V(\sigma, \pi)$ separates $u$ from $z$. Therefore $V(\sigma, \pi)$ must contain a point of $S-\bar{Y}$. But $V(\sigma, \pi) \subset X_{Q+1} \subset \bar{Y}$. So it follows that $\bar{Y}$ contains a point in $S-\bar{Y}$. This is clearly a contradiction. Hence, $S=\bar{Y}$. Thus we have that $S$ is separable.

The next theorem can be viewed as an extension of Theorem 1.3 and a theorem due to J. H. Roberts [7], which he discovered in answer to a question of L. B. Treybig [12]. While obtained independently, the method of proof is somewhat similar to that of Roberts. First we need a lemma.

LEMMA 4.3. If in a connected metric space for every two points $p$ and $q$, every open set containing $p$ contains a continuum which separates $p$ from $q$, then each component of the complement of a continuum is open $\left({ }^{28}\right)$.

Proof. Suppose that $C$ is a continuum lying in a connected metric space $S$ and let $q$ be a point in $C$. Now let $p$ be a point in $S-C$, and $E$ be a component of $S-C$ containing $p$. Let $n$ be a positive integer such that $C \cdot D_{1 / n}(p)=0$, and $F$ be a continuum lying in $D_{1 / n}(p)$ such that $F$ separates $p$ from $q$. Then there are mutually separate open sets $H$ and $K$ such that $p \in H, q \in K$, and $H+K=S-F$. Since $C$ is connected and $q \in C, K \supset C$. Therefore, $H+F \subset S-C$. We shall show that $H+F$ is connected. Suppose, on the contrary, that $H+F$ is not connected and let $J$ and $L$ be two mutually separate sets such that $J+L=H+F$. Since $F$ is connected, we can let $L \supset F$. Now $J$ has no limit point in $K$, nor in $L$. And, dually, $K$ has no limit point in $J$, nor does $L$. But $S-J=K+L$. It follows that $S$ is the sum of two mutually separate sets $J$ and $K+L$, that is, $S$ is not connected. This is a contradiction. Hence,

$\left({ }^{27}\right)$ See Theorem (10.1) on p. 15 of G. T. Whyburn [13]. The reader is cautioned that in that book the terms "compact" and "continuum" have stronger meanings than those used here.

$\left({ }^{28}\right)$ This is a well-known result. We include a proof only for the convenience of the reader. 
$H+F$ is connected. Consequently $H+F \subset E$, which means that there is an open set, namely $H$, which contains $p$ and lies in $E$. Therefore $E$ is an open set. Thus we have that each component of $S-C$ is an open set.

THEOREM 4.4. If $S$ is a connected metric space such that (i) no point separates $S$, (ii) if $p$ and $q$ are two points in $S$, then every region containing $p$ contains a continuum which separates $p$ from $q$, (iii) there is a separable closed set which separates $S$, and (iv) every separable closed set which separates $S$ contains two points which are separated by a separable closed set (this includes (i)), then the space $S$ is separable $\left({ }^{29}\right)$.

Proof. For each two points $p$ and $q$ which are separated by a separable closed set, let $\gamma(p, q)$ be the least positive integer $\delta$ such that $D_{1 i \delta}(p)$ is separated from $D_{1 / \delta}(q)$ by a separable closed set, and let $V(p, q)$ be a separable closed set separating $D_{1 / \gamma(p, q)}(p)$ from $D_{1 / \gamma(p, q}(q)$. Now let it be that $A_{0}$ is a separable closed set which separates $S$ (by (iii)), $B_{0}$ is a set to which a point $p$ belongs only in case $p$ is in $A_{0}$ and $A_{0}$ contains a point $q$ which is separated from $p$ by a separable closed set (by (iv)), $C_{0}$ is a countable dense subset of $B_{0}, E_{0}$ and $F_{0}$ are functions such that for each point $p$ in $C_{0}, E_{0}(p)$ is a set to which a point $q$ belongs only in case $q$ is in $A_{0}$ and $q$ is separated from $p$ by a separable closed set, and $F_{0}(p)$ is a countable dense subset of $E_{0}(p)$, and let $G_{0}=A_{0}+\sum_{p \in C_{0}} \sum_{q \in F_{0}(p)} V(p, q)$. Clearly $G_{0}$ is separable. Furthermore, since $A_{0} \subset G_{0}$, there are two points in $G_{0}$ which are separated by a separable closed set. Hence, if $n$ is a nonnegative integer, let $A_{n+1}=\bar{G}_{n}$ and define $B_{n+1}, C_{n+1}, E_{n+1}, F_{n+1}$, and $G_{n+1}$ in the appropriate manner. Let $H=\sum_{n=0}^{\infty} A_{n}$. The set $\bar{H}$ is a separable closed set. We shall show that $S=\bar{H}$. Suppose, on the contrary, that $\bar{H} \neq S$.

Let $J$ be a nondegenerate component of $S-\bar{H}$. If $\bar{H} \subset \bar{J}$, then $\bar{H} \cap \bar{J}$ contains two points, for example in $A_{0}$, which are separated by a separable closed set. If $\bar{H} \notin \bar{J}$, let $U$ be a component of $S-\bar{J}$. $U$ is open, by Lemma 4.3. Let $K$ be the boundary of $U . K \subset \bar{J}$ and $K$ contains at least two points. If there is a point of $K$ in $J$, let $p$ be such a point and $q$ be another point in $K$. Let $L$ be a continuum lying in $S-\bar{H}$ which separated $p$ from $q$. It follows that $L \cap U \neq 0$ and $L \cap J \neq 0$, which contradicts the fact that $J$ is a component of $S-\bar{H}$. Hence $K \subset \bar{H} \cap \bar{J}$, and so, $K$ is a separable closed set which separates $U$ from $S-\bar{U}$. Therefore, by (iv) $K$, and hence $\bar{H} \cap \bar{J}$, contains two points which are separated by a separable closed set. Hence we can let $x$ and $y$ be two points in $\bar{H} \cap \bar{J}$ which are separated by a separable closed set. Let $\sigma_{0}$ and $\pi_{0}$ be points in $H$ such that $d\left(x, \sigma_{0}\right)<1 / 4 \gamma(x, y)$ and $d\left(y, \pi_{0}\right)$ $<1 / 4 \gamma(x, y)$ and $N$ be a positive integer such that each of $\sigma_{0}$ and $\pi_{0}$ is in $A_{N}$. It follows that $V(x, y)$ separates $\sigma_{0}$ from $\pi_{0}$. Therefore, $\sigma_{0}$ is in $B_{N}$ and we can let $\sigma_{1}$ be a point in $C_{N}$ such that $d\left(\sigma_{0}, \sigma_{1}\right)<1 / 4 \gamma(x, y)$. Then $V(x, y)$ separates $\sigma_{1}$ from $\pi_{0}$.

$\left({ }^{29}\right)$ The theorem in its present form was suggested by the referee as being the proposition actually shown by a proof in the original draft. Though that is not the case, the theorem itself turns out to be true. I am most grateful to the referee for his acumen. 
Therefore, $\pi_{0}$ is in $E_{N}\left(\sigma_{1}\right)$ and we can let $\pi_{1}$ be a point in $F_{N}\left(\sigma_{1}\right)$ such that $d\left(\pi_{0}, \pi_{1}\right)$ $<1 / 4 \gamma(x, y)$. Now we have that $x \in D_{1 / 2 \gamma(x, y)}\left(\sigma_{1}\right) \subset D_{1 / \gamma(x, y)}(x)$, and $y \in D_{1 / 2 \gamma(x, y)}\left(\pi_{1}\right)$ $\subset D_{1 / \gamma(x, y)}(y)$ and $V(x, y)$ separates $D_{1 / 2 \gamma(x, y)}\left(\sigma_{1}\right)$ from $D_{1 / 2 \gamma(x, y)}\left(\pi_{1}\right)$. By definition, $\gamma\left(\sigma_{1}, \pi_{1}\right)<2 \gamma(x, y)$, and hence $V\left(\sigma_{1}, \pi_{1}\right)$ separates $D_{1 / 2 \gamma(x, y)}\left(\sigma_{1}\right)$ from $D_{1 / 2 \gamma(x, y)}\left(\pi_{1}\right)$. It follows that $V\left(\sigma_{1}, \pi_{1}\right)$ separates $x$ from $y$. Hence, $V\left(\sigma_{1}, \pi_{1}\right)$ contains a point in $J$, which contradicts the fact that $V\left(\sigma_{1}, \pi_{1}\right) \subset G_{N} \subset \bar{H}$. Therefore, $\bar{H}=S$. Thus we have that $S$ is separable.

The next theorem follows from Theorem 4.4.

THEOREM 4.5 (ROBERTS [7]). If $S$ is a connected metric space such that (i) no point separates $S$, and (ii) if $p$ and $q$ are two points in $S$, then every region containing $p$ contains a separable continuum which separates $p$ from $q$, then the space $S$ is separable.

2. The locally connected situation. In the preceding section we omitted the hypothesis of local connectedness. Here we inquire about possible generalizations of Theorem 1.2, where the hypothesis of local connectedness is assumed. Theorem 4.7 is such a generalization.

DEFINITION 4.1. A space $S$ is countably-semi-locally-connected at a point $p$ means that if $U$ is an open set containing $p$ then $U$ has an open subset $V$ which contains $p$ and $S-U$ intersects at most countably many components of $S-V$.

THEOREM 4.6. If $S$ is a connected metric space such that (i) $S$ is countably-semilocally-connected at each point which separates $S$, (ii) $S$ is locally connected, (iii) there is a nondegenerate $\left({ }^{30}\right)$ separable closed set which separates $S$, and (iv) every nondegenerate separable closed set which separates $S$ contains two points which are separated by a separable closed set, then $S$ is separable.

Proof. If $p$ is a point and $n$ is a positive integer, let $\sigma(p, n)$ be the least positive integer $\delta$-in case such a $\delta$ exists-for which there is an open set $W$ such that $D_{1 / \delta}(p) \subset W \subset D_{1 / n}(p)$ and $S-D_{1 / n}(p)$ intersects at most countably many components of $S-W$-and in this case let $W(p, n)$ be one such $W$ so that $D_{1 / \sigma(p, n)}(p)$ $\subset W(p, n) \subset D_{1 / n}(p)$, and let $Z(p, n)$ be a countable set which intersects each of the countable components of $S-W(p, n)$ that $S-D_{1 / n}(p)$ intersects. Clearly, if there is a point which separates $S, \sigma(p, n)$ and $W(p, n)$ can be defined for that $p$ and any sufficiently large positive integer $n$. Define $\gamma, V, A_{0}, B_{0}, C_{0}, E_{0}, F_{0}$, and $G_{0}$ as in the proof of Theorem 4.4. Now let $X_{0}=$ a countable dense subset of $G_{0}$, and let $Y_{0}=G_{0}+\sum_{p \in X_{0}} \sum_{m=1}^{\infty} Z(p, m)$, for those $(p, m)$ for which $Z$ can be defined. The set $Y_{0}$ is separable and $Y_{0}$ contains some two points, for example in $A_{0}$, which are separated by a separable closed set. Hence, if $n$ is a nonnegative integer, let $A_{n+1}=\bar{Y}_{n}$, and define $B_{n+1}, C_{n+1}, E_{n+1}, F_{n+1}, G_{n+1}, X_{n+1}$, and $Y_{n+1}$ in the

$\left({ }^{30}\right)$ A set is degenerate means that the set consists of only one point. A set is nondegenerate means that it is not degenerate. 
appropriate manner. Now letting $H=\sum_{n=0}^{\infty} A_{n}$, we have that $\bar{H}$ is a separable closed set. We shall show that $\bar{H}=S$.

Suppose, on the contrary, that $S \neq \bar{H}$. Let $J$ be a component of the open set $S-\bar{H}$, and $K$ be the boundary of $J$. Since $S$ is locally connected, $J$ is an open set and $K \subset \bar{H}$.

Case 1 . $K$ consists of only one point $p$. It follows that $p$ separates $J$ from $S-(J+p)$. Let $n$ be a positive integer such that $D_{1 / n}(p)$ does not contain $J$. Let $r$ be a point in $H$ such that $d(p, r)<1 / 4 \sigma(p, 4 n)$, and $N$ be a positive integer such that $r \in A_{N}$. Let $t$ be a point in $X_{N}$ such that $d(r, t)<1 / 4 \sigma(p, 4 n)$. We now have the following relations:

$$
p+t \subset D_{1 / 2 \sigma(p, 4 n)}(t) \subset D_{1 / \sigma(p, 4 n)}(p) \subset W(p, 4 n) \subset D_{1 / 4 n}(p) \subset D_{1 / 2 n}(t) \subset D_{1 / n}(p) .
$$

Hence, by definition, $\sigma(t, 2 n) \leqq 2 \sigma(p, 4 n)$. Also, $D_{1 / 2 n}(t)$ does not contain $J$. Let $q$ be a point in $J \cdot\left(S-D_{1 / 2 n}(t)\right)$ and let $T$ be the component of $S-W(t, 2 n)$ such that $q \in T$. Since $p$ separates the open set $J$ from $S-(J+p)$, it must be that $T \subset J$. Therefore, $Z(t, 2 n)$ contains a point of $T$, which contradicts the fact that $Z(t, 2 n)$ $\subset A_{N+1} \subset H$. Therefore $\bar{H}=S$.

Case 2. The set $K$ is nondegenerate. In this case an argument similar to that in the last paragraph of the proof of Theorem 4.4 shows that $S=\bar{H}$.

Thus we have that the space $S$ is a separable space.

A simply stated, but quite general, corollary of Theorem 4.6 is the following condition for separability which appears in [10].

THEOREM 4.7. If no point separates a connected, locally connected, metric space $S$, and every two points are separated by a separable closed set, then $S$ is separable.

3. Remarks and examples. In Theorem 4.1 condition (ii) cannot be relaxed by requiring the connected set to be only separable. The space $\Gamma$ in Part II shows this. Notice that the space $\Gamma$ is also locally peripherally compact.

Condition (ii) in Theorem 4.3 does not necessarily imply that the space is compact-continuum-wise connected. The following is an example of a space which satisfies condition (ii) of Theorem 4.3, but is not compact-continuum-wise connected.

Let $R$ be the set of rational numbers in $[0,1]$. Let $R=R_{1}+R_{2}$, where $R_{1}$ and $R_{2}$ are mutually exclusive and each of them is dense in $[0,1]$. Let $\left\{R_{11}, R_{12}, R_{13}, \ldots\right\}$ be an infinite sequence of subsets of $R_{1}$, each dense in $[0,1], R_{1 i} \cdot R_{1 j}=0$ for $i \neq j$, and $\sum_{i=1}^{\infty} R_{11}=R_{1}$. Let $\left\{R_{21}, R_{22}, R_{23}, \ldots\right\}$ be a similar decomposition of $R_{2}$. The space consists of the following points in the plane:

$$
\begin{aligned}
\{(x, y) \mid & 0 \leqq x \leqq 1, y=0\}+\{(x, y) \mid 0 \leqq x \leqq 1, y=1\} \\
& +\sum_{i=1}^{\infty}\left\{(x, y) \mid x \in R_{1 i}, 0 \leqq y \leqq 1-1 / i\right\}+\sum_{i=1}^{\infty}\left\{(x, y) \mid x \in R_{2 i}, 1 / i \leqq y \leqq 1\right\} .
\end{aligned}
$$

The topology of the space is the relative plane topology. 
We do not know whether in Theorem 4.4 the condition (i) can be replaced by the condition (i) in Theorem 4.6.

For a measure of the sharpness of the other theorems in this chapter, we again appeal to the space $\Gamma$ described in Part II. It can be easily seen that many other attempts to weaken the conditions given in this chapter are defeated by the properties of the space $\Gamma$.

As an application of Theorem 4.7, one may observe that any metric space satisfying Axioms $0-4$ of R. L. Moore's Foundations of point set theory [6] will be separable.

\section{REFERENCES}

1. Paul Alexandroff, Uber die Metrization der im kleinen kompakten topologische Räume, Math. Ann. 92 (1924), 294-301.

2. E. E. Grace and R. W. Heath, Separability and metrizability in pointwise paracompact Moore spaces, Duke Math. J. 31 (1964), 603-610. MR 29 \#6464.

3. F. B. Jones, A theorem concerning locally peripherally separable spaces, Bull. Amer. Math. Soc. 41 (1935), 437-439.

4. J. R. Isbell, Uniform spaces, Math. Surveys, no. 12, Amer. Math. Soc., Providence, R. I., 1964. MR 30 \#561.

5. L. F. McAuley, A relation between perfect separability, completeness, and normality in semi-metric spaces, Pacific J. Math. 6 (1956), 315-326. MR 18, 325.

6. R. L. Moore, Foundations of point set theory, Amer. Math. Soc. Colloq. Publ., vol. 13, Amer. Math. Soc., Providence, R. I., 1932.

7. J. H. Roberts, A problem of Treybig concerning separable spaces, Duke Math. J. 28 (1961), 153-155. MR 24 \#A1705.

8. Prabir Roy, Separability of connected, locally connected metric spaces, Duke Math. J. 29 (1962), 99-100. MR 24 \#A3627.

9. - Separability of metric spaces, Doctoral Dissertation, University of North Carolina, Chapel Hill, N. C., 1961.

10. L. B. Treybig, Concerning certain locally peripherally separable spaces, Pacific J. Math. 10 (1960), 697-704. MR 22 \#7104.

11. - Concerning local separability in locally peripherally separable spaces, Proc. Amer. Math. Soc. 10 (1959), 957-958. MR 22 \#224.

12. —- Separability in metric spaces, Duke Math. J. 27 (1960), 383-386. MR 24 \#A1704.

13. G. T. Whyburn, Analytical topology, Amer. Math. Soc. Colloq. Publ., vol. 28, Amer. Math. Soc., Providence, R. I., 1942. MR 4, 86.

\section{RUTGERS UNIVERSITY,}

New Brunswick, New Jersey 08903

State University OF NeW York,

Binghamton, New YoRK 13901 